\title{
The psychiatric consequences
}

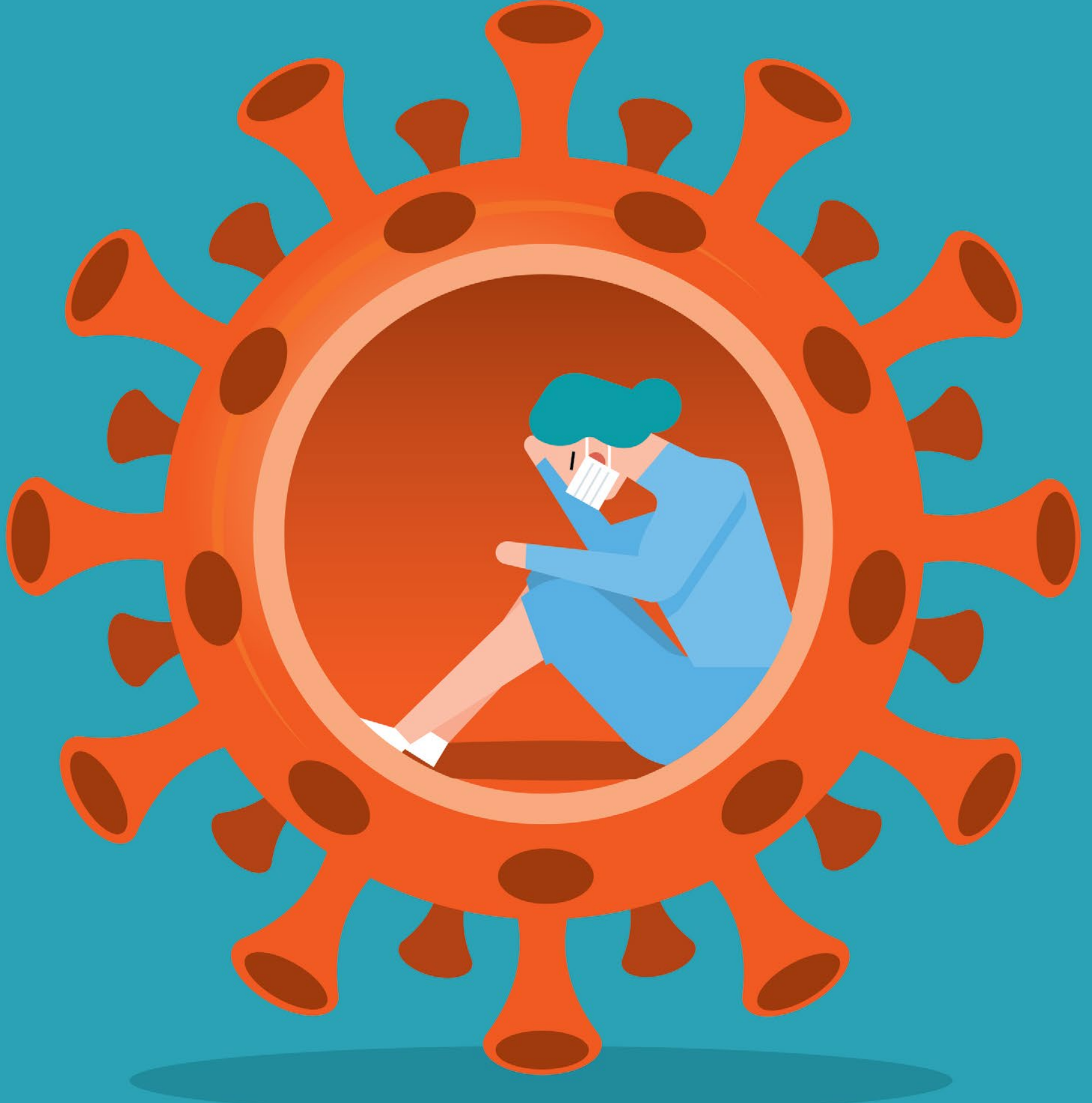




\section{of COVID-19: 8 Studies}

\section{Initial research explores the toll on health care workers, patients, and the general public}

\author{
Sy Atezaz Saeed, MD, MS \\ Professor and Chair \\ Department of Psychiatry and \\ Behavioral Medicine \\ East Carolina University Brody School of Medicine \\ Greenville, North Carolina
}

\section{Karim Hebishi, MD}

PGY-3 Internal Medicine/Psychiatry Resident

Department of Internal Medicine

Department of Psychiatry and

Behavioral Medicine

East Carolina University Brody School of Medicine

Greenville, North Carolina

\section{Disclosures}

The authors report no financial relationships with any companies whose products are mentioned in this article, or with manufacturers of competing products.

doi: $10.12788 /$ cp. 0055 evere acute respiratory syndrome coronavirus 2 (SARS-CoV-2), the novel coronavirus that is causing the ongoing coronavirus disease 2019 (COVID-19) pandemic, was first reported in late 2019. ${ }^{1}$ As of mid-October 2020, >39 million confirmed cases of COVID-19 had been reported worldwide, and the United States was the most affected country with $>8$ million confirmed cases. ${ }^{2}$ Although the reported symptoms of COVID-19 are primarily respiratory with acute respiratory distress syndrome, SARS-CoV-2 has also been shown to affect other organs, including the brain, and there are emerging reports of neurologic symptoms due to COVID-19. ${ }^{3}$

Psychological endurance will be a challenge that many individuals will continue to face during and after the pandemic. Physical and social isolation, the disruption of daily routines, financial stress, food insecurity, and numerous other potential triggers for stress response have all been intensified due to this pandemic, creating a situation in which many individuals' mental well-being and stability is likely to be threatened. The uncertain environment is likely to increase the frequency and/or severity of mental health problems worldwide. Psychiatric symptoms such as anxiety and depression have been reported among patients with SARS-CoV-1 during the previous severe acute respiratory syndrome (SARS) epidemic. ${ }^{4}$

In this article, we summarize 8 recent studies, systematic reviews, and meta-analyses to provide an overview of the psychiatric consequences of

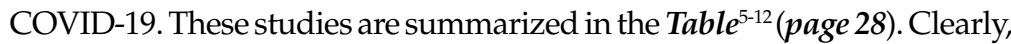
the studies reviewed here are preliminary evidence, and our understanding of COVID-19's effects on mental health, particularly its long-term sequelae, is certain to evolve with future research. However, these 8 studies describe how COVID-19 is currently affecting mental health among health care workers, patients, and the general public. 


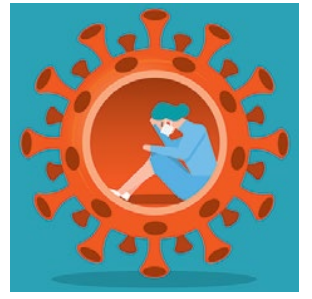

Psychiatric toll of COVID-19

\section{Clinical Point}

\section{Compared with the general public, health care workers had poor sleep quality and more obsessive- compulsive symptoms}

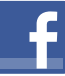

Discuss this article at www.facebook.com/ MDedgePsychiatry
1. Vindegaard N, Benros ME. COVID-19 pandemic and mental health consequences: systematic review of the current evidence. Brain Behav Immun. 2020;89:531-542.

Vindegaard and Benros ${ }^{5}$ conducted a systematic review of the literature to characterize the impact of COVID-19-related psychiatric complications and COVID19 's effect on the mental health of patients infected with COVID-19, as well as noninfected individuals.

\section{Study design}

- This systematic review included 43 studies that measured psychiatric disorders or symptoms in patients with COVID-19 and in a non-infected group.

- The non-infected group consisted of psychiatric patients, health care workers, and the general population.

- The review excluded studies with participants who were children, adolescents, or older adults, or had substance abuse or somatic disorders.

\section{Outcomes}

- Only 2 studies included patients with confirmed COVID-19 infection. Of the remaining 41 studies, 2 studies examined the indirect effects of the pandemic on psychiatric patients, 20 studies examined health care workers, and 19 studies examined the general population. Eighteen of the studies were case-control studies and 25 had no control group

Patients with confirmed COVID-19 infection. One case-control study showed an increased prevalence of depression in patients with COVID-19 who had recently recovered $(29.2 \%)$ compared with participants who were in quarantine $(9.8 \%)$. The other study showed posttraumatic stress symptoms in $96 \%$ of hospitalized patients with COVID-19 who were stable.

Patients with preexisting psychiatric disorders. Two studies found increased symptoms of psychiatric disorders.

Health care workers. Depression (6 studies) and anxiety symptoms (8 studies) were increased among health care workers compared with the general public or administrative staff. However, 2 studies found no difference in these symptoms among health care workers compared with the general public. Poor sleep quality and more obsessive-compulsive symptoms were reported in health care workers compared with the general public.

General public. Compared to before the COVID-19 pandemic, lower psychological well-being and increased rates of depression and anxiety were noted among the general public. Higher rates of anxiety and depression were also found in parents of children who were hospitalized during the pandemic compared with prior to the pandemic. One study found no difference between being in quarantine or not.

- Current or prior medical illness was associated with higher rates of anxiety and depression. One study found higher social media exposure was associated with increased anxiety and depression. Female health care workers had higher rates of anxiety and depression symptoms.

\section{Conclusions/limitations}

This systematic review included 39 studies from Asia and 4 from Europe, but none from other continents, which may affect the external validity of the results. Most of the studies included were not case-controlled, which limits the ability to comment on association. Because there is little research on this topic, only 2 of the studies focused on psychiatric symptoms in patients with COVID-19. In most studies, the reporting of psychiatric disorders was vague and only a few studies used assessment tools, such as the General Anxiety Disorder-7 or the Patient Health Questionnaire-9, for reporting depression and anxiety.

2. Pappa S, Ntella V, Giannakas T, et al. Prevalence of depression, anxiety, and insomnia among healthcare workers during the COVID-19 pandemic: a systematic review and meta-analysis. Brain Behav Immun. 2020;88:901-907.

Pappa et $\mathrm{al}^{6}$ examined the effects of the COVID-19 pandemic on the mental health of health care workers, with specific focus on the prevalence of anxiety, depression, and insomnia. 


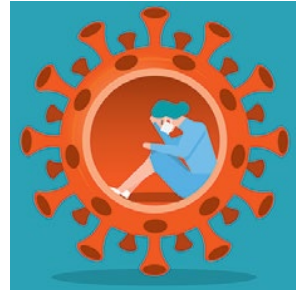

Psychiatric toll of COVID-19

\section{COVID-19's psychiatric consequences: Health care workers, patients, and the general public}

\begin{tabular}{l:l} 
Study & Design \\
\hdashline $\begin{array}{l}\text { Vindegaard } \\
\text { and Benros }\end{array}$ & Review of 43 studies that \\
(2020) & evaluated the psychiatric \\
& effect of COVID-19 on infected \\
& patients, patients with pre- \\
& existing mental illness, health \\
& care workers, and the general \\
& public
\end{tabular}

Pappa et $\mathrm{al}^{6}$ Meta-analysis of 13 studies (2020) of the prevalence of anxiety, depression, and insomnia in health care workers

\section{Clinical Point \\ Increased rates of anxiety and depression were noted among the general public}

Loades et $\mathrm{al}^{7}$ Systematic review of 63 (2020) studies examining the effect of loneliness/social isolation on the mental health of children and adolescents

Outcomes

COVID-19 infection caused increasing posttraumatic stress symptoms and depression. There were also increased rates of anxiety, depressive symptoms, poor sleep quality, and obsessive-compulsive symptoms among health care workers and the general public. Female gender and preexisting medical conditions were risk factors. Two studies reported worsening psychiatric symptoms in patients with preexisting mental illness

There was a pooled prevalence of $23.2 \%$ for anxiety (female $=29 \%$, male $=20.9 \%$ ), $22.8 \%$ for depression (female $=26.87 \%$, male $=20.3 \%$ ) and $38.9 \%$ for insomnia. Female participants showed higher rates of anxiety and depression. No subgroup analysis was performed for insomnia There was a positive association between social isolation/loneliness in childhood/adolescence and

Rogers et $\mathrm{al}^{8}$ Systematic review and (2020) random-effects model metaanalysis of 65 studies of the psychiatric effects of 3 severe coronavirus-associated epidemics (SARS, MERS, and COVID-19)

Shiozawa Systematic review of 10 et $\mathrm{al}^{9}(2020)$ articles that examined psychiatric issues associated with COVID-19

\begin{tabular}{|l|l|}
$\begin{array}{l}\text { Salari et } \text { al }^{10} \\
\text { (2020) }\end{array}$ & $\begin{array}{l}\text { Meta-analysis of } 17 \\
\text { observational studies } \\
\text { examining the prevalence } \\
\text { of anxiety and stress in the } \\
\text { general population during the } \\
\text { COVID-19 pandemic }\end{array}$ \\
\hline $\begin{array}{l}\text { Preti et al } \\
\text { (2020) }\end{array}$ & $\begin{array}{l}\text { Systematic review of } 44 \\
\text { studies looking at the } \\
\text { psychological impact of } \\
\text { epidemics and outbreaks on } \\
\text { health care workers }\end{array}$
\end{tabular}

Varatharaj et Cross-sectional analysis of the $\mathrm{al}^{12}(2020)$ prevalence of psychiatric and neurologic complications in patients with COVID-19 across multiple centers in the United Kingdom
Insomnia was found in $34 \%$ to $36.1 \%$ of health care workers, and severe anxiety symptoms were found in $45 \%$. Variable results were found for PTSD and depression. Female gender, being a nurse, and working on high-risk units were risk factors for worse psychological outcomes

Sixty-two percent of patients had cerebrovascular events, $31 \%$ had altered mental status, and $5 \%$ had peripheral neurologic disorders. Of those with altered mental status, 7 patients had encephalitis, 9 had unspecified encephalopathy, and 23 had a psychiatric diagnosis as classified by a psychiatrist or neuropsychiatrist. Ten patients had new-onset psychosis and 2 had exacerbation of preexisting mental illness. However, no pre-defined criteria were used to measure the reported diagnoses 


\section{Study design}

- Researchers searched for studies on PubMed, Medline, and Google Scholar. A random effect meta-analysis was used on the included 13 cross-sectional studies with a total of 33,062 participants. Twelve of the included studies were conducted in China and 1 in Singapore.

- Evaluation of the risk of bias of included studies was assessed using a modified form of the Newcastle-Ottawa Scale (NOS), with a score $>3$ considered as low risk of bias.

\section{Outcomes}

- Results were categorized by gender, rating scales, severity of depression, and professional groups for subgroup analysis.

- The primary outcomes were prevalence $(\mathrm{p})$, confidence intervals $(\mathrm{CI})$, and percentage prevalence $(\mathrm{p} \times 100 \%)$. Studies with a low risk of bias were sub-analyzed again $(n=9)$.

- Anxiety was evaluated in 12 studies, depression in 10 studies, and insomnia in 5 studies (all 5 studies had a low risk of bias).

- There was a pooled prevalence of $23.2 \%$ for anxiety $(29 \%$ female, $20.9 \%$ male), $22.8 \%$ for depression $(26.87 \%$ female, $20.3 \%$ male), and $38.9 \%$ for insomnia. Female participants showed higher rates of anxiety and depression, while no subgroup analysis was performed for insomnia.

- The subgroup analysis of pooled data after excluding each study showed that no single study had $>2 \%$ effect on the pooled analysis.

- The subgroup analysis by gender, professional group, and severity suggested that there was an increased prevalence of anxiety and depression in female health care workers, which was consistent with the increased prevalence in the general population.

\section{Conclusions/limitations}

There was a questionable effect of betweenstudy heterogeneity. Different studies used different rating scales and different cutoff points on the same scales, which might make the results of pooled analysis unreliable, or might be assumed to increase the confidence. Despite the use of different scales and cutoff points, there was still a high prevalence of anxiety, depression, and insomnia. All studies were conducted in a single geographical region (12 in China and 1 in Singapore). None of the included studies had a control group, either from the general population or compared with preCOVID-19 rates of depression, anxiety, and insomnia in health care workers.

\section{Loades ME, Chatburn E, Higson-Sweeney} $\mathrm{N}$, et al. Rapid systematic review: the impact of social isolation and loneliness on the mental health of children and adolescents in the context of COVID-19 [published online June 3, 2020]. J Am Acad Child Adolesc Psychiatry. 2020;50890-8567(20)30337-3. doi: 10.1016/j. jaac.2020.05.009.

The COVID-19 pandemic has led to long periods of isolation/quarantine, social distancing, and school closures, all which have resulted in significant upheaval of the lives of children and adolescents. Loades et $\mathrm{al}^{7}$ explored the impact of loneliness and disease-containment measures related to the COVID-19 pandemic on children and adolescents.

\section{Study design}

- Researchers conducted a systematic review of 63 studies examining the impact of loneliness or disease-containment measures on healthy children and adolescents. located through a search of Medline, PsycINFO, and Web of Science. Sixty-one studies were observational, and 2 were interventional.

- The search yielded studies published between 1946 and March 29, 2020.

- The quality of studies was assessed using the National Institutes of Health quality assessment tool.

\section{Outcomes}

- Results by mental health symptom or disorder were categorized as follows:

Depression. Forty-five studies examined depressive symptoms and loneliness; only 6 studies included children age $<10$. Most reported a moderate to large correlation

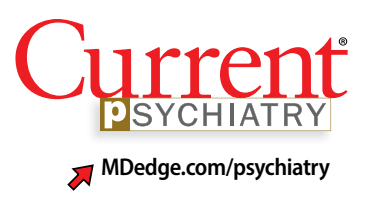

Clinical Point

In 1 review, the pooled prevalence of anxiety among health care workers was $23 \%$ 


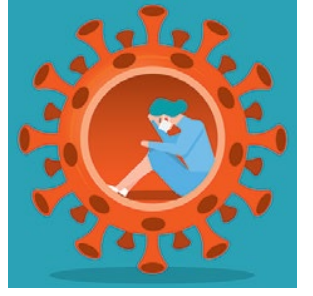

Psychiatric toll of COVID-19

\section{Clinical Point \\ Children who experienced enforced quarantine were 5 times more likely to require treatment for posttraumatic stress}

$(0.12 \leq \mathrm{r} \leq 0.81)$, and most of them included a measure of depressive symptoms. The association was stronger in older and female participants. Loneliness was associated with depression in 12 longitudinal studies that followed participants for 1 to 3 years. However, 3 studies ( 2 in children and 1 in adolescents) found no association between loneliness and depression at follow-up.

Anxiety. Twenty-three studies examined symptoms of anxiety and found a small to moderate correlation between loneliness/social isolation and anxiety $(0.18 \leq \mathrm{r}$ $\leq 0.54$ ), with duration of loneliness being more strongly associated with anxiety than intensity of loneliness. However, social anxiety or generalized anxiety were associated more with loneliness $([0.33 \leq \mathrm{r} \leq 0.72]$ and $[\mathrm{r}=0.37,0.40]$, respectively). Three longitudinal studies found associations between loneliness and subsequent anxiety, and 1 study did not find an association between loneliness at age 5 and increased anxiety at age 12.

Mental health and well-being. Two studies found negative associations between social isolation/loneliness and well-being and mental health.

\section{Conclusions/limitations}

There is decent evidence of a strong association between loneliness/social isolation in childhood/adolescence and the development of depression, with some suggestion of increased rates in females. However, there was a small to moderate association with anxiety with increased rates in males. The length of social isolation was a strong predictor of future mental illness. Children who experienced enforced quarantine were 5 times more likely to require mental health services for posttraumatic stress symptoms.

The compiled evidence presented in this study looked at previous similar scenarios of enforced social isolations; however, it cannot necessarily predict the effect of COVID-19-associated social distancing measures. Most of the studies included were cross-sectional studies and did not control for confounders. Social isolation in childhood or adolescence may be associated with developing mental health problems later in life and should be considered when implementing school closures and switching to online classes. Loades et $\mathrm{al}^{7}$ suggested that the increased rate of electronic communication and use of social media in children and adolescents may mitigate this predicted effect of social isolation.

4. Rogers JP, Chesney E, Oliver D, et al. Psychiatric and neuropsychiatric presentations associated with severe coronavirus infections: a systematic review and metaanalysis with comparison to the COVID-19 pandemic. Lancet Psychiatry. 2020;7(7): 611-627.

To identify possible psychiatric and neuropsychiatric implications of the COVID-19 pandemic, Rogers et $\mathrm{al}^{8}$ examined 2 previous coronavirus epidemics, SARS and Middle East respiratory syndrome (MERS), and COVID-19.

\section{Study design}

- Researchers conducted a randomeffects model meta-analysis and systematic review of 65 studies and 7 preprints from 10 countries, including approximately 3,559 case studies of psychiatric and neuropsychiatric symptoms in participants infected with the 3 major coronavirus-induced illnesses (SARS, MERS, and COVID-19).

- Pure neurologic complications and indirect effects of the epidemics were excluded.

- The systematic review followed PRISMA guidelines.

- The quality of the studies was assessed using the NOS.

\section{Outcomes}

- Outcomes measured were psychiatric signs or symptoms; symptom severity; diagnoses based on ICD-10, DSM-IV, the Chinese Classification of Mental Disorders (third edition), or psychometric scales; quality of life; and employment.

- Results were stratified as acute or post-illness:

Acute illness. Delirium was the most frequently reported symptom in all 3 


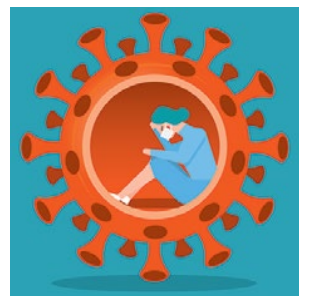

Psychiatric toll of COVID-19

\section{Clinical Point}

Despite the high incidence of psychiatric symptoms in previous coronavirus infections, it was hard to draw conclusions for COVID-19 continued from page 30

coronavirus infections. Depression, anxiety, or insomnia were also reported in MERS and SARS infections. Mania was described in SARS, but it was almost entirely present in cases treated with highdose corticosteroids, which are not used routinely for COVID-19.

Post-illness. There was increased incidence of depression, anxiety, fatigue, and posttraumatic stress disorder (PTSD) in the post-illness stage of previous coronavirus epidemics (SARS and MERS), but there was no control group for comparison. There was not enough data available for COVID-19.

\section{Conclusions/limitations}

Three studies were deemed to be of high quality, 32 were low quality, and 30 were moderate quality. Despite the high incidence of psychiatric symptoms in previous coronavirus infections, it was difficult to draw conclusions due to a lack of adequate control groups and predominantly low-quality studies. The difference in treatment strategies, such as the use of high-dose corticosteroids for MERS and SARS, but not for COVID-19, made it difficult to accurately predict a response for COVID-19 based on previous epidemics.

\section{Shiozawa $P$, Uchida RR. An updated systematic review on the coronavirus pandemic: lessons for psychiatry. Braz J Psychiatry. 2020;42(3):330-331.}

Schiozawa et $\mathrm{al}^{9}$ conducted a systematic review of articles to identify psychiatric issues during the COVID-19 pandemic.

\section{Study design}

- Researchers conducted a systematic review of 10 articles ( 7 articles from China, 1 from the United States, 1 from Japan, and 1 from Korea) that described strategies for coping with the COVID-19 pandemic and/or provided a descriptive analysis of the clinical scenario, with an emphasis on psychiatric comorbidities.

- The study used PRISMA guidelines to summarize the findings of those 10 studies. There were no pre-set outcomes or inclusion criteria.

\section{Outcomes}

- The compiled results of the 10 studies showed high rates of new-onset insomnia, anxiety, and relapse of underlying conditions such as depression.

- One study found increased hospital visits and misinterpretations of any symptom in patients with health anxiety (health anxiety was not defined).

- One study found some benefit from multidisciplinary psychological care and online counseling for both patients and health care workers.

\section{Conclusions/limitations}

Because each of the 10 studies examined extremely different outcomes, researchers were unable to compile data from all studies to draw a conclusion.

6. Salari N, Hosseinian-Far A, Jalali R, et al. Prevalence of stress, anxiety, depression among the general population during the COVID-19 pandemic: a systematic review and meta-analysis. Global Health. 2020;16(1):57.

Salari et $\mathrm{al}^{10}$ examined the prevalence of stress, anxiety, and depression in the general population during the COVID-19 pandemic.

\section{Study design}

- Researchers conducted a systematic review and meta-analysis of 17 observational studies examining the prevalence of anxiety and stress in the general population during the COVID-19 pandemic. The STROBE checklist was used to assess the quality of studies.

- Only studies judged as medium to high quality were included in the analysis.

\section{Outcomes}

- The prevalence of stress was $29.6 \%$ (5 studies, sample size 9,074 individuals).

- The prevalence of anxiety was 31.9\% (17 studies, sample size 63,439 individuals).

- The prevalence of depression was $33.7 \%$ (14 studies, sample size of 44,531 individuals).

- A sub-analysis of rates by continent revealed that Asia had highest prevalence of anxiety and depression (32.9\% and 
$35.3 \%$, respectively). Europe had the highest rates of stress $(31.9 \%)$.

\section{Conclusions/limitations}

There is an increased prevalence of anxiety, stress, and depression in the general population amid the COVID-19 pandemic. None of the included studies compared rates to before the pandemic. Most studies used online surveys, which increased the chance of sample bias. Most studies originated from China and Iran, which had the highest rates of infection when this review was conducted.

\section{Preti E, Di Mattei V, Perego G, et al. The} psychological impact of epidemic and pandemic outbreaks on healthcare workers: rapid review of the evidence. Curr Psychiatry Rep. 2020;22(8):43.

Preti et al ${ }^{11}$ performed a review of the literature to determine the impact of epidemic/pandemic outbreaks on health care workers' mental health.

\section{Study design}

- Researchers conducted a rapid systematic review of 44 studies examining the psychological impact of epidemic/ pandemic outbreaks on health care workers.

- Of the 44 studies, $27(62 \%)$ referred to the SARS outbreak, $5(11 \%)$ referred to the MERS outbreak, $5(11 \%)$ referred to the COVID-19 outbreak, $3(7 \%)$ referred to the influenza A virus subtype H1N1 outbreak, $3(7 \%)$ referred to the Ebola virus disease outbreak, and $1(2 \%)$ referred to the Asian lineage avian influenza outbreak.

\section{Outcomes}

- During these outbreaks, insomnia was found in $34 \%$ to $36.1 \%$ of health care workers, and severe anxiety symptoms in $45 \%$.

- The prevalence of PTSD-like symptoms among health care workers during the outbreaks was $11 \%$ to $73.4 \%$. Studies of the COVID-19 pandemic reported the highest prevalence of PTSD-like symptoms $(71.5 \%$ to $73 \%)$. After 1 to 3 years following an outbreak, $10 \%$ to $40 \%$ of health care workers still had significant PTSDlike symptoms.
- Anxiety was reported in $45 \%$ of health care workers during the COVID-19 pandemic.

- A sub-analysis revealed a positive association between anxiety, PTSD, and stress symptoms and being female gender, being a nurse, and working on high-risk units.

- Perceived organizational support and confidence in protective measures were negatively associated with psychological symptoms.

\section{Conclusions/limitations}

Lessons from previous outbreaks and early data from the COVID-19 pandemic suggest that health care workers experience higher levels of psychological symptoms during outbreaks. Findings of this study suggest that organizational support and confidence in protective measures can mitigate this effect. To help preserve the well-being of health care workers, adequate training should be provided, appropriate personal protective equipment should be readily available, and support services should be well established.

8. Varatharaj A, Thomas N, Ellul MA, et al. Neurological and neuropsychiatric complications of COVID-19 in 153 patients: a UK-wide surveillance study. Lancet Psychiatry. 2020;7(10):875-882.

Varatharaj et $\mathrm{al}^{12}$ conducted a surveillance study in patients in the United Kingdom to understand the breadth of neurologic complications of COVID-19.

\section{Study design}

- Researchers performed a cross-sectional analysis of the prevalence of psychiatric and neurologic complications in patients with COVID-19 across multiple centers in United Kingdom. Data were collected through the anonymous online reporting portals of several major neurology and psychiatric associations. Retrospective reporting was allowed.

- Evidence of SARS-CoV-2 infection was defined as:

Confirmed CoVID-19 (114 cases) if polymerase chain reaction (PCR) of respiratory

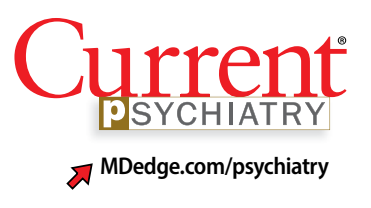

Clinical Point

Female gender, being a nurse, and working on high-stress units increase the risk of worse psychological outcomes 


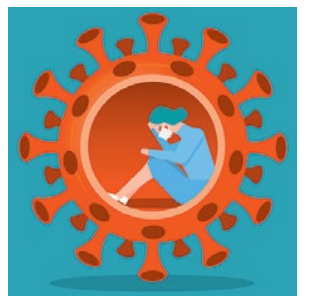

Psychiatric toll of COVID-19

\section{Clinical Point}

A review and analysis
of 17 observational
studies found an
increased prevalence
of anxiety, stress, and
depression among
the general public

A review and analysis studies found an increased prevalence depression among the general public

\section{Related Resources}

- Ryznar E. Evaluating patients' decision-making capacity during COVID-19. Current Psychiatry. 2020;19(10):34-40.

- Freudenreich O, Kontos N, Querques J. COVID-19 and patients with serious mental illness. 2020;19(9):24-27,33-39.

- Esterwood E, Saeed SA. Past epidemics, natural disasters, COVID19, and mental health: learning from history as we deal with the present and prepare for the future [published online August 16, 2020]. Psychiatr Q. 2020:1-13. doi: 10.1007/s11126-020-09808-4.

samples (eg, nasal or throat swab) or CSF was positive for viral RNA or if serology was positive for anti-SARS-CoV-2 immunoglobulin M (IgM) or immunoglobulin G (IgG).

Probable COVID-19 (6 cases) if a chest radiograph or chest $\mathrm{CT}$ was consistent with COVID-19 but PCR and serology were negative or not performed.

Possible COVID-19 (5 cases) if the disease was suspected on clinical grounds by the notifying clinician, but PCR, serology, and chest imaging were negative or not performed.

\section{Outcomes}

- Sixty-two percent of patients presented with cerebrovascular events (intracerebral hemorrhage, ischemic stroke, vasculitis, or other). Thirty-one percent of patients presented with altered mental status (AMS), and 5\% had peripheral neurologic disorders.
- Of those with AMS, 18\% (7 patients) had encephalitis, 23\% (9 patients) had unspecified encephalopathy, and 59\% (23 patients) had a psychiatric diagnosis as classified by the notifying psychiatrist or neuropsychiatrist. Ten patients (43\%) of the 23 patients with neuropsychiatric disorders had new-onset psychosis, while only 2 patients had an exacerbation of a preexisting mental illness.

\section{Conclusions/limitations}

This study had an over-representation of older adults. There was no control group for comparison, and the definition of confirmed COVID-19 included a positive IgM or IgG without a positive PCR or chest imaging. Although all psychiatric conditions reported were confirmed by a psychiatrist or neuropsychiatrist, there were no pre-defined criteria used for reported diagnoses.

\footnotetext{
References

1. Huang C, Wang Y, Li X, et. al. Clinical features of patients infected with 2019 novel coronavirus in Wuhan, China. Lancet. 2020;395(10223):497-506.

2. John Hopkins University \& Medicine. Coronavirus Resource Center. 2020. https://coronavirus.jhu.edu. Accessed October 16, 2020.

3. Montalvan V, Lee J, Bueso T, et al. Neurological manifestations of COVID-19 and other coronavirus infections: a systematic review. Clin Neurol Neurosurg. 2020;194:105921.

4. Wu P, Fang Y, Guan Z, et al. The psychological impact of the SARS epidemic on hospital employees in China: exposure, risk perception, and altruistic acceptance of risk. Can J Psychiatry. 2009;54(5):302-311.

5. Vindegaard N, Benros ME. COVID-19 pandemic and mental health consequences: systematic review of the current evidence. Brain Behav Immun. 2020;89:531-542.

6. Pappa S, Ntella V, Giannakas T, et al. Prevalence of depression, anxiety, and insomnia among healthcare
}

\section{Bottom Line}

Evidence from studies of previous outbreaks and early data from the coronavirus disease 2019 (COVID-19) pandemic suggest that during outbreaks, health care workers experience higher levels of psychological symptoms than the general population. There has been an increased prevalence of anxiety, stress, poor sleep quality, obsessive-compulsive symptoms, and depression among the general population during the pandemic. COVID-19 can also impact the CNS directly and result in delirium, cerebrovascular events, encephalitis, unspecified encephalopathy, altered mental status, or peripheral neurologic disorders. Patients with preexisting psychiatric disorders are likely to have increased symptoms and should be monitored for breakthrough symptoms and acute exacerbations. 
workers during the COVID-19 pandemic: a systematic review and meta-analysis. Brain Behav Immun. 2020;88: 901-907.

7. Loades ME, Chatburn E, Higson-Sweeney N, et al Rapid systematic review: the impact of social isolation and loneliness on the mental health of children and adolescents in the context of COVID-19 [published online June 3, 2020]. J Am Acad Child Adolesc Psychiatry. 2020;S0890-8567(20)30337-3. doi: 10.1016/j. jaac.2020.05.009.

8. Rogers JP, Chesney E, Oliver D, et al. Psychiatric and neuropsychiatric presentations associated with severe coronavirus infections: a systematic review and metaanalysis with comparison to the COVID-19 pandemic Lancet Psychiatry. 2020;7(7):611-627.
9. Shiozawa P, Uchida RR. An updated systematic review on the coronavirus pandemic: lessons for psychiatry. Braz J Psychiatry. 2020;42(3):330-331.

10. Salari N, Hosseinian-Far A, Jalali R, et al. Prevalence of stress, anxiety, depression among the general population during the COVID-19 pandemic: a systematic review and meta-analysis. Global Health. 2020;16(1):57.

11. Preti E, Di Mattei V, Perego G, et al. The psychological impact of epidemic and pandemic outbreaks on healthcare workers: rapid review of the evidence [published online July 10, 2020]. Curr Psychiatry Rep. 2020;22(8):43.

12. Varatharaj A, Thomas N, Ellul MA, et al. Neurological and neuropsychiatric complications of COVID-19 in 153 patients: a UK-wide surveillance study. Lancet Psychiatry. 2020;7(10):875-882.

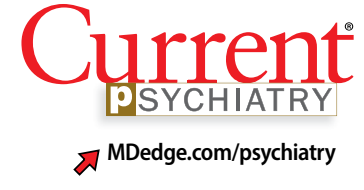

Clinical Point

COVID-19 can impact the CNS directly and result in delirium, cerebrovascular events, and altered mental status 